\title{
Exploring Ethnic Group and Geographic Differences in Social Axioms in the USA
}

\author{
Theodore M. Singelis, Dharm P. S. Bhawuk, William K. Gabrenya Jr., \\ Michele Gelfand, Jake Harwood, Pa Her, Junko Tanaka-Matsumi, \\ and Joseph Vandello
}

\begin{abstract}
This study investigates the dimensionality of a recently developed measure of social beliefs - the Social Axioms Survey (SAS) for American respondents. Ethnic group and geographical differences in the endorsement of social beliefs were also assessed with the SAS with samples of college and noncollege students in eight locations in the USA $(N=2,164)$. Results of exploratory and confirmatory factor analyses supported the five-factor structure found previously in international samples (Leung \& Bond, 2004). Differences among ethnic groups showed that African Americans scored higher on the belief dimension of religiosity than did Asian or Caucasian Americans. Asian Americans were more inclined toward socially cynical beliefs than were other ethnic groups and believed more in fate control than did Caucasian or Hispanic Americans. Differences in social beliefs across locations were limited to religiosity beliefs when only Caucasian American respondents were considered. Implications for comparisons of samples from the USA with other countries are discussed.
\end{abstract}

\author{
T.M. Singelis $(\varangle)$ \\ Department of Psychology, California State University, Chico, CA 95929-0234 \\ e-mail: tsingelis@csuchico.edu \\ D.P.S. Bhawuk \\ University of Hawai'i, Honolulu, Hawai'i USA \\ W.K. Gabrenya \\ Florida Institute of Technology, Melbourne, Florida USA \\ M. Gelfand \\ University of Maryland, Baltimore, Maryland USA \\ J. Harwood \\ University of Arizona, Tucson, Arizona USA \\ P. Her \\ Virginia Tech, Blacksburg, Virginia USA \\ J. Tanaka-Matsumi \\ Kwansei Gakuin University, Nishinomiya-City, Japan \\ J. Vandello \\ University of South Florida, Tampa, Florida USA
}


A persistent problem in cross-cultural research has been finding ways to describe cultures in ways that allow for comparison among them. The most common cultural comparison uses the individualism-collectivism dimension (IC) (Hofstede, 1980; Triandis, 1995) and self-construal as the individual-level or psychological indicator (Markus \& Kitayama, 1991; Singelis, 1994). Among all the dimensions available, none has generated the research that has accrued to the IC dimension (see Oyserman, Coon, \& Kemmelmeier, 2002 for a review and critique). In an effort to add to the cultural dimensions available to scholars wishing to compare and understand cultures, an SAS was recently developed (Leung et al., 2002). The dimensions identified in the survey are based on beliefs rather than values or selfconstruals.

The results of this initial study suggested that five social axioms factors may be universal: Fate Control, Reward for Application, Cynicism, Religiosity, and Social Complexity. The pan-cultural dimensionality was confirmed in a larger, subsequent study (Leung \& Bond, 2004). The purpose of the current study was to explore ethnic group and geographical variations in social beliefs with the SAS in a population from the USA. In addition, we sought to assess the dimensionality of the SAS in this population with a large enough sample to ensure the stability of the factor structure obtained.

\section{Values and Beliefs}

One of the most widely cited systems for describing cultures is Hofstede's (1980) four-dimensional framework based on work-related values in 40 countries. Of the four original dimensions (individualism-collectivism, masculinity-femininity, power distance, and uncertainty avoidance) only individualism-collectivism has been widely used as a theoretical framework. The Chinese Culture Connection (1987) conducted a study based on Eastern values in which they added a distinctive dimension, "Confucian Work Dynamism" (now called "long-term orientation"), to Hofstede's original four. Another widely recognized system of cultural dimensions comes from Schwartz (1992, 1994), who researched 41 cultural groups from 39 nations, establishing a mapping of cultures along seven culturelevel domains of values.

Although the value perspective has been influential, there need to be additional dimensions by which we can understand cultural variations. According to Leung et al. (2002) additional frameworks serve two purposes: "They may yield information about cultural variations that cannot be detected by the value perspective and/ or they may provide needed triangulation for well-known results based on values." (p. 287)

In addition to previous constructs studied by cultural scholars, general beliefs may serve as a complementary framework to values, since beliefs have been shown to relate to a variety of social behaviors (Fraser \& Gaskell, 1990; Furnham, 1988). For example, researchers have found that beliefs are related to moral reasoning and moral conduct (Brody \& Shaffer, 1982). Locus of control, a general belief about 
the cause of events that happen to oneself, has been shown to relate to a variety of behaviors (e.g., Spector, 1982). Trustworthiness, a general belief about human nature, has been related to various interpersonal behaviors (Wrightsman, 1992).

\section{Social Axioms Survey}

We shall not discuss the background of the Social Axioms Survey at length since this material is covered well elsewhere in this volume. Briefly, Social Axioms are generalized beliefs about personhood, the social and physical environment, or the spiritual world. Social beliefs are instrumental in coping with problems of survival and effective functioning, and the dimensions across which beliefs vary should be universal and identifiable in different cultural groups and serve the four major functions of attitudes: "They facilitate the attainment of important goals (instrumental), help people protect their self-worth (ego defensive), serve as a manifestation of people's value (value-expressive), and help people understand the world (knowledge)" (Leung et al., 2002, p. 288).

A scale based solely on belief statements was constructed by Leung et al. (2002). Using data collected in Hong Kong, Venezuela, USA, Germany, and Japan five factors were established: Social Cynicism: items representing a negative view of human nature and social relations; Reward for Application: items representing a general belief that effort, knowledge, and careful planning will lead to positive results; Social Complexity: items declaring that there are no rigid rules, but rather multiple ways of achieving a given outcome, and that inconsistency in human behavior is common; Fate Control: items represent a belief that life events are predetermined and that there are ways for people to influence these outcomes; and Spirituality (later renamed Religiosity): items that refer to the reality of supernatural forces and the attest to the positive functions of religion (Leung et al., 2002).

Additional studies have supported both the structure and the validity of these original five dimensions of social beliefs (Leung \& Bond, 2004; Singelis, Hubbard, Her, \& An, 2003).

\section{Ethnic Variations in the USA}

A body of research provides evidence for ethnic variations within the USA on cultural dimensions, although the work in this area is neither systematic nor conclusive (see Oyserman et al., 2002). For example, Asian Americans were significantly higher than European Americans in collectivism (Singelis, Triandis, Bhawuk, \& Gelfand, 1995) and were more interdependent and less independent than Euro Americans (e.g., Singelis, 1994; Singelis \& Brown, 1995).

Research has also suggested that there are other differences between Euro Americans and African Americans (Baldwin \& Hopkins, 1990; Jones, 1988; Triandis, 1994). Euro Americans are more concerned with what they own as a deter- 
minant of identity (e.g., "I am a student"), whereas African Americans emphasize what they express in personal style and movement and "how I appear to others" (Triandis, 1994). African American culture can be best described as emphasizing spirituality, harmony with nature, present orientation, expressiveness, and collectivism (Boykin, 1983; Jones, 1988). Conversely, Euro Americans have been described as materialistic/mechanistic, future orientated, controlled in expression, individualist, and high achieving. For a more extensive discussion of African and Euro American differences see Jones (1988) and Boykin (1983). At the same time, Majors and Mancini-Billson (1993) argue that African Americans have internalized the white male values of self-reliance and economic success, despite structural barriers, and thus differences in beliefs about reward for application may be small.

\section{Regional Variations in Cultural Dimensions}

Using data aggregated by state, Vandello and Cohen (1999) created a collectivism index to measure the differences of individualism and collectivism among the 50 American states. The final index consisted of eight factors relating to family structure, living arrangements, social, political, religious, and economic practices. The 50 states were aggregated into nine cultural regions: Confederate (Deep) South; Peripheral South; Northeast; New York and New Jersey; Midwest and Great Lakes; Mountain West and Great Plains; Southwest; Utah; and Hawaii. Significant regional differences were found in the state-level collectivism index. Hawaii was more collectivist than all other regions; the Mountain West and Great Plains region were more individualist than all other regions; and the Deep South was significantly more collectivist than the Mountain West and Great Plains, the Great Lakes and Midwest, and the Northeast. Although past research has characterized the USA as particularly high on individualism, the results of Vandello and Cohen (1999) suggest that there are regional variations in psychological collectivism within the USA corresponding to these state-level differences in collectivism. Given these findings, it is expected that social beliefs will vary by region.

\section{Method}

\section{Participants}

A total of 2,164 students and nonstudents from eight locations in the USA participated in the study. There were more students $(1,274)$ than nonstudents $(886)$ and more women $(1,166)$ than men $(979)$. Four participants did not report their student status and 19 did not indicate their sex. Age was reported in categories: less than 20 (753 or $34.8 \%$ ); 21 to 30 (755 or $34.9 \%) ; 31$ to 40 (170 or $7.9 \%) ; 41$ to $50(307$ or $14.4 \%)$; 51 to 60 (131 or $6.1 \%$ ); and 60 and over (46 or $2.1 \%$ ). Two 
Table 1 Distribution of ethnic backgrounds

\begin{tabular}{lrc}
\hline Ethnic background & Frequency & Percent \\
\hline Black & 59 & 2.7 \\
African American & 48 & 2.2 \\
Caucasian & 1,416 & 65.4 \\
Chinese & 130 & 6.0 \\
East Indian & 18 & 0.8 \\
Fillipino & 41 & 1.9 \\
Hawaiian & 10 & 0.5 \\
Hispanic & 41 & 1.9 \\
Japanese & 96 & 4.4 \\
Korean & 37 & 1.7 \\
Latino/a & 18 & 0.8 \\
Mexican & 24 & 1.1 \\
Native American & 7 & 0.3 \\
Puerto Rican & 17 & 0.8 \\
Samoan & 4 & 0.2 \\
Vietnamese & 14 & 0.6 \\
Other & 168 & 7.8 \\
Missing & 18 & 0.8 \\
Total & 2,164 & 100 \\
\hline
\end{tabular}

participants did not indicate their age. The majority of participants self-identified as Caucasian, constituting $65.4 \%$ of the sample, although other people from other groups described themselves by using a variety of identifications (see Table 1).

\section{Instruments}

Social Axioms Survey Because this study was conducted early in the development of the SAS (Leung et al., 2002), an 82 item version of the scale was used and included interpersonal harmony items. Five-point, Likert-type response formats were anchored by "strongly disbelieve" and "strongly believe."

Demographic Items In addition to the scales, demographic probes were also included. The respondents were asked to list: age group, sex, education, and occupation. Another section asked additional questions about the respondent's background, such as ethnicity, place of residence, birth-place, political affiliation, and religion.

\section{Procedure}

The Survey was administered in eight different locations: Chico, California; Lawrence, Kansas; College Park, Maryland; Los Angeles, California; New York, New York; Melbourne, Florida; Honolulu, Hawaii; and Urbana-Champaign, Illinois. The survey, which contained a battery of instruments, took approximately $30 \mathrm{~min}$ 
to complete. Some of the student participants received extra credit for their participation. Some of the nonstudent participants were recruited through a snowball technique where student participants collected data from nonstudents.

\section{Results}

\section{Structure of Social Axioms}

To determine if the structure of the SAS was similar to that found previously, a two-step process was undertaken. The 82 social axioms items were first submitted to an exploratory factor analysis with half the sample, and then a follow-up confirmatory factor analysis was undertaken with the remaining half.

Exploratory Factor Analysis Half of the sample $(\underline{n}=1,084)$ was randomly selected for the principal components analysis of 82 social axioms items using a varimax rotation. Based on the scree plot, a six-factor solution accounting for $28.20 \%$ of the variance was chosen. Items were examined for their fit. Criteria for item selection were a primary loading of at least 0.35 and a secondary loading less than 0.20. No items composing the sixth factor met these criteria, so the final exploratory analysis yielded 40 items on 5 factors (see Table 2 for items and factors). It should be noted that the sixth factor that was dropped due to insufficient item loadings was predominantly made up of interpersonal harmony items. These data support the elimination of this factor in the development of the finalSAS.

Confirmatory Factor Analysis The cases not used in the exploratory analysis above were used for the confirmatory analysis $(\underline{n}=1,080)$ by EQS version 5 (Bentler, 1995). Items were set to load exclusively on the factors designated in the exploratory analysis. Factors were set to be uncorrelated. The chi-square was significant $(2,459.27$ with 725 $d f, p<.001$ ). Goodness-of-fit indexes were moderate (Bentler-Bonnett normed fit $=0.71$; Lisrel adjusted goodness of fit $=0.87$ ). The standardized root mean square residual was acceptable (0.059) and the root means square error of approximation was quite good (0.047) with a $90 \%$ confidence interval of 0.045 to 0.049 (see Hu \& Bentler, 1999). All items loaded significantly on their designated factors. See Table 2 for item loadings.

We concluded that the five-factor solution is a good representation of the social axioms data in our American sample. Items on each factor were averaged to give scores for each dimension of beliefs. The CFA and the lack of substantial correlations between the dimensions indicate that these are distinct dimensions. See Table 3 for correlations across dimensions and alpha coefficients.

\section{Variations in Social Axioms}

Sex Differences A MANOVA was used for this and all of the following analyses due to the small, but significant, correlations between the social axioms dimensions 
CFA loading

Factor 1: Cynicism $\alpha=.77$

1. Power and status make people arrogant 0.61

2. Powerful people tend to exploit others $\quad 0.57$

3. Kind-hearted people are easily bullied $\quad 0.50$

4. People will stop working hard after they secure a comfortable life $\quad 0.49$

5. Too much money ruins one's character $\quad 0.47$

6. Kind-hearted people usually suffer losses $\quad 0.47$

7. Caring about societal affairs only brings trouble for yourself $\quad 0.47$

8. Old people are usually stubborn and biased $\quad 0.44$

9. People deeply in love are usually blind $\quad 0.44$

10. It is rare to see a happy ending in real life $\quad 0.39$

11. It is hard to make friends with people who have different opinions $\quad 0.39$ from yourself

12. Young people are impulsive and unreliable

13. If one belongs to a marginal group, it is difficult to gain acceptance from the majority group

\section{Factor 2: Religiosity $\alpha=.81$}

1. Religious faith contributes to good mental health $\quad 0.77$

2. Belief in a religion helps one understand the meaning of life 0.76

3. There is a supreme being controlling the universe $\quad 0.67$

4. Belief in a religion makes people good citizens 0.58

5. Religious people are more likely to maintain moral standards 0.54

6. One feels safer in the world through a belief in a supreme being 0.49

7. Religion makes people escape from reality $\quad-0.45$

8. After life on earth, one carries on an existence in another form 0.41

9. All things in the universe have been determined 0.40

\section{Factor 3: Reward for Application $\alpha=.61$}

1. Honesty is a pre-requisite for a happy life 0.54

2. One will succeed if he/she really tries 0.51

3. Hard working people will achieve more in the end 0.51

4. A harmonious family life leads to career success 0.43

5. Knowledge is necessary for success 0.41

$\begin{array}{ll}\text { 6. Every problem has a solution } & 0.37\end{array}$

Factor 4: Social Complexity $\alpha=.63$

1. True partnership can only exist when there is mutual respect 0.61

2. A pleasant interpersonal environment and a sense of well-being lead to better performance $\quad 0.49$

3. Human behavior changes with the social context $\quad 0.46$

4. One has to deal with matters according to the specific circumstances 0.42

5. A modest person can make a good impression on people 0.39

6. One's behaviors may be contrary to his or her true feelings $\quad 0.37$

7. People may have opposite behaviors on different occasions 0.36

Factor 5: Fate Control $\alpha=.58$

1. Fate determines one's successes and failures $\quad 0.53$

2. Good luck follows if one survives a disaster $\quad 0.51$

3. Individual characteristics, such as appearance and date of birth, affect
one's fate

4. There are certain ways to help us improve our luck and avoid unlucky things $\quad 0.40$

5. There are many ways for people to predict what will happen in the future $\quad 0.34$ 
Table 3 Correlations and alphas

\begin{tabular}{lcllll}
\hline Social axioms dimension & 1 & 2 & 3 & 4 & 5 \\
\hline 1. Cynicism & 0.77 & & & & \\
2. Religiosity & 0.01 & 0.81 & & & \\
3. Reward for Application & 0.04 & $0.27^{* * *}$ & 0.61 & & \\
4. Social Complexity & -0.01 & $0.08^{* * *}$ & $0.27^{* * *}$ & 0.63 & \\
5. Fate Control & $0.27^{* * * *}$ & $0.07^{* * *}$ & $0.10^{* * *}$ & -0.02 & 0.58 \\
\hline Note: Significance levels are: $0.01^{* *}, 0.001^{* * *}$. & & \\
Reliabilities are reported on the diagonal. & & &
\end{tabular}

(see Table 3). A significant Wilks' Lambda for sex was found $(F[5,1996]=11.97$, $p<.001)$. Between-subjects' effects indicated that men $(M=2.72)$ were more socially cynical than women $(M=2.58)(F=7.73, p<.001, d=.28)$, while women $(M=3.31)$ reported higher beliefs on the religiosity dimension than men $(M=3.17)$ $(F=8.94, p<.001, d=.20)$. Women $(M=4.11)$ were also higher in social complexity than men $(M=4.06)(F=1.45, p<.01, d=.13)$. No differences in reward for application or fate control were found. Due to the power of such a large sample to make small differences significant, effect sizes are noted above and in analyses that follow. The sizes of these sex differences ( $d$ from 0.13 to 0.28 ) are relatively small (Cohen, 1988). In subsequent analyses possible interactions with sex were checked and none were found.

Differences by Ethnic Group To investigate the effects of ethnicity on social beliefs another MANOVA was performed with self-reported ethnicity as the independent variable. Because there were many groups represented (see Table 1) and we wanted to keep the groups large enough for statistical power, data were collapsed across some groups after first checking to determine if differences in their social beliefs existed. No differences were found between subjects selfidentifying as Chinese, Japanese, Korean, and Vietnamese, so they were combined to form an Asian group $(n=300)$. No differences were found between participants who self-identified as African American or Black, so they were combined as well $(n=99)$. Finally, no differences were found between those self-identifying as Hispanic, Latino/a, Mexican, or Puerto Rican, so they were combined into a Hispanic group $(n=89)$. The last group in this analysis is those who identified themselves as Caucasian $(n=1,336)$. It should be noted that the combining of groups for the current analysis in no way implies that they are equivalent or culturally identical in ways other than their social beliefs as measured in this study.

Wilks' Lambda for ethnic group was significant $(F[15,5013]=10.59$, $p<.001)$. Between-subjects' effects indicated that ethnic group was significant for all of the social axiom dimensions except social complexity: religiosity $F$ ( 3 , $1820)=6.33, p<.001, R^{2}=.01$; fate control $F(3,1820)=18.80, p<.001, R^{2}=.03$; reward for application $F(3,1820)=4.77, p<.01, R^{2}=.008$; and social cynicism $F(3,1820)=33.63, p<.001, R^{2}=.053$. Post hoc analyses using Tukey's Honest Significant Difference tests with significance level set at 0.05 were done to determine the significant difference between the four ethnic groups. Means for 
Table 4 Means by ethnic group

\begin{tabular}{llllll}
\hline Ethnic Group & Religiosity & $\begin{array}{l}\text { Reward for } \\
\text { Application }\end{array}$ & $\begin{array}{l}\text { Social } \\
\text { Complexity }\end{array}$ & $\begin{array}{l}\text { Fate } \\
\text { Control }\end{array}$ & Cynicism \\
\hline African American $(n=99)$ & $3.49^{\mathrm{b}}$ & $3.75^{\mathrm{a}}$ & $4.08^{\mathrm{a}}$ & $2.62^{\mathrm{ab}}$ & $2.70^{\mathrm{a}}$ \\
Asian American $\left(n \_=300\right)$ & $3.18^{\mathrm{a}}$ & $3.77^{\mathrm{a}}$ & $4.10^{\mathrm{a}}$ & $2.79^{\mathrm{b}}$ & $2.88^{\mathrm{b}}$ \\
Caucasian $(n=1,336)$ & $3.22^{\mathrm{a}}$ & $3.65^{\mathrm{a}}$ & $4.10^{\mathrm{a}}$ & $2.50^{\mathrm{a}}$ & $2.57^{\mathrm{a}}$ \\
Hispanic American $(n=89)$ & $3.36^{\mathrm{ab}}$ & $3.76^{\mathrm{a}}$ & $4.15^{\mathrm{a}}$ & $2.56^{\mathrm{a}}$ & $2.62^{\mathrm{a}}$ \\
\hline
\end{tabular}

Note: Means within columns that have the same superscript are not significantly different by Tukey HSD post hoc tests $(p<.05)$.

the ethnic groups are reported in Table 4. Briefly, African Americans reported higher Religiosity than Asian Americans and Caucasian Americans, but did not significantly differ from Hispanic Americans. Asian Americans were more likely to believe in Fate Control than Caucasians or Hispanics, although the Asian group did not differ from the African Americans. Finally, Asian Americans scored higher on the Social Cynicism dimension than the other three groups who did not differ. Readers should note that although the analysis of variance for reward for application showed a significant effect for ethnic group, the post hoc Tukey HSD test, which uses the harmonic mean of the unbalanced group sizes, returned the result of no differences between the groups.

Regional Differences Data were collected in a number of locations, but many of the participants in each location were not native to that location. Because cultural transmission takes place during the socialization process, we reasoned the best test of regional culture would be to compare people who were born and raised in a particular place. Drawing on the demographic data collected, we selected those who reported having been born and raised in the same state. Overall there were 1,483 who fit this criterion. To have sufficient power to detect differences, we eliminated people from states having fewer than 100 participants who were born and raised there. We were left with 1,017 participants from five states: California $(n=255)$, Hawaii $(n=124)$, Illinois $(n=213)$, Kansas $(n=149)$, and New York $(n=276)$. However, given the prior analysis of ethnicity and the fact that ethnic group was confounded with states where participants were born and raised $\left(\chi^{2}(12)=572.64, p<.0001\right)$, we decided to use only those who self-identified as Caucasian in our analysis of variation across location. This further restriction resulted in participants from the following states being included in the current analysis: California $(n=174)$, Illinois $(n=183)$, Kansas $(n=135)$, and New York $(n=207)$.

A MANOVA was performed with state as the between-subjects' variable. Wilks' Lambda for state was significant in this analysis $(F[15,1908]=2.58$, $p<.001)$. Between-subjects' effects indicated that state was a significant effect only for the dimension of Religiosity, $F(3,695)=8.30, \underline{p}<.001, R^{2}=.03$. Post hoc analysis with Tukey's Honest Significant Difference test $(p<.05)$ revealed that participants from California scored lower on the religiosity dimension than those from Kansas and Illinois. 


\section{Discussion}

This study sought to establish the dimensionality of the SAS in the USA, and to explore ethnic group and geographic differences in social beliefs. The data from the two factor analyses offer support for the five-factor model of social axioms in the USA. The low inter-factor correlations further support the independence of these five dimensions. One problem that remains is the relatively low reliabilities of some dimensions. The lack of strong internal consistency has been a persistent problem with measures of cultural difference (see Oyserman et al., 2002). Nonetheless, all items contributed significantly in the confirmatory factor analysis.

The SAS was born of, and is primarily intended for, cross-cultural/cross-ethnic studies, so the breadth of the items allows sufficient range to provide validity in cultural systems with somewhat different emic aspects. In other words, what is sacrificed in internal consistency increases the range of items, making them more appropriate for measuring the construct in a variety of cultures (see Singelis et al., 1995). Still, those wishing to use this instrument may wish to explore the possibility of adding additional items in a given cultural setting to bolster the internal consistency of the belief dimensions measured.

A second purpose of this study was to explore ethnic differences. It must be noted that the differences between ethnic groups that were found, although significant, had small effect sizes. Even though the effect sizes were small, they support the ability of the SAS to detect cultural differences and indicate that this new measure may prove useful as an additional tool for scholars wishing to explore cultural variation. Overall, we found ethnic differences on four of the five dimensions, although conservative post hoc analyses revealed significant differences in only three. Specifically, social cynicism, endorsing a negative view of human nature and social institutions, varied between the groups-Asian Americans being more cynical than Caucasian Americans, Hispanic Americans and African Americans who did not differ. Consistent with past research (e.g., Grantham, 1994; Reed, 1972, 1983), African Americans held stronger beliefs about religion than Asian Americans and Caucasian Americans, but did not significantly differ from Hispanic Americans. Asian Americans, who did not differ from the African Americans, were more likely to believe in fate control than Caucasian or Hispanic Americans. One speculation is that this result points to the influence of East Asian religions such as Confucianism and Buddhism on Asian Americans (Slote \& De Vos, 1998). Certainly an unsophisticated understanding of the concept of karma, although more germane to the Hindu religion, might lead to the belief that aspects of one's life are predetermined. It may also be that the experience of immigration, which is common among Asian-American families, has contributed to this sense of fate control. Confirming Asian-American belief in fate control and exploring its source are areas in need of additional research.

Some might speculate that more than cultural influences, political and power factors should affect people's social beliefs. In other words, a person who is a minority, as opposed to a member of the dominant group, might tend to be more 
socially cynical and to believe more in fate control because of their relative powerlessness in the social and political order. Minority status might also affect one's belief in reward for application, although one could make a good argument in either direction. On one hand, a minority person might believe that barriers are too great and that application makes no difference. On the other hand, a different minority person may see the same barriers and believe that the only way to overcome them is to apply one's self, a strategy that will then be rewarded. To examine these highly speculative ideas, data were collapsed into dominant (Caucasian Americans) and minority (Hispanic and African Americans) groups. Asian Americans were not used, since most in this sample were from Hawai'i where Asians are not a minority. Analyses showed that the minority group was, in fact, higher on the dimensions of Social Cynicism, Fate Control, and Reward for Application, although the differences were quite small. It would be interesting to do some qualitative analyses to determine the relative influences of culture and minority status on social beliefs. While there may be a combination of influences for a given individual, it seems more likely that the social beliefs of some individuals are highly influenced by their cultural background and socialization, while the beliefs of others are highly influenced by their minority status and the experiences associated with it.

It was expected that individuals in different locations would differ in their social beliefs. This expectation was largely unfulfilled. Due to the unbalanced distribution of ethnic groups and birthplaces in the samples, an analysis was undertaken in which ethnicity was restricted to Caucasian and the location was designated as the place where subjects were born and raised. In this analysis, Religiosity was the only social belief that differed across location, and that difference was limited to those in California scoring lower on religiosity than participants from Illinois and Kansas. Overall these analyses lead to the conclusion that there is a general agreement in social beliefs among Caucasians in our sample, no matter where they were born and raised in the USA.

Importantly, this finding contrasts with the results of Vandello and Cohen (1999). Regional variations may occur when accounting for their operationalization of collectivism, but we have found little empirical support for variation in social beliefs. The emphasis in Vandello and Cohen (1999) was on data defined at the state level, whereas data in the current study are analyzed at the individual level. This difference points to the possible discrepancy between ecological and individual-level correlations, an issue in the study of beliefs addressed by Leung and Bond (2008). Another factor that may have contributed to our failure to find regional differences is the fact that we did not include a sample from the Deep South in our analysis. We sampled in Florida, but when we took into account where people were born and raised, that state dropped out of the analysis. Since the Deep South seemed to be the most different mainland region in the Vandello and Cohen (1999) study, it may be that there are differences in social beliefs in that region not measured in the current study. Further, it should be noted that many other locations sampled in Vandello and Cohen (1999) were not considered in the present study.

Taken together, the results validate the SAS and are consistent with research demonstrating its dimensionality and sensitivity to cultural differences (Bond, 2001; 
Leung et al., 2002). The SAS has already proven to be a useful tool in predicting behaviors from beliefs in Hong Kong (Bond, Leung, Au, Tong, \& ChemongesNielson, 2004). We believe that it will also prove useful in the USA. For instance, further research may be conducted to determine how these five dimensions affect helping behavior, job preferences, or child rearing practices.

Although there were few significant regional variations in social axioms within the USA when ethnicity was controlled, there are some implications that may be drawn from the results. We suggest that there may be a uniformity of social beliefs among Caucasian Americans in the USA. This fact has implications for scholars comparing the results of a single sample from the USA with a sample from another country, as is common in cross-cultural research. If, as indicated here, there is a mostly uniform culture among Caucasian Americans in the USA, such single-sample comparisons may be warranted regardless of where in the USA the sample is drawn.

However, researchers must take care in ensuring that ethnicity in the USA sample is controlled. On the other hand, one may argue justifiably that a homogeneous sample which controls ethnicity is not representative of the USA. This tension raises the question of who are we talking about when we say, for example, Americans are more individualistic than Koreans? Scholars must consider their answer carefully when engaging in cross-cultural comparisons.

\section{References}

Baldwin, J \& Hopkins, R. (1990). African American and European-American cultural differences as assessed by the worldviews paradigm: An empirical analysis. Western Journal of Black Studies, 14, 38-52.

Bentler, P. M. (1985-1995). EQS. Encino, CA: Multivariate Software.

Bond, M. H., Leung, K., Au. A., Tong, K. K., \& Chemonges-Nielson, Z. (2004). Combining social axioms with values in predicting social behaviors. European Journal of Personality, 18, 177-191.

Boykin, A. W. (1983). The academic performance of Afro-American children. In J. Spence (Ed.), Achievement and achievement motives. New York: Freeman.

Brody, G., \& Shaffer, D. (1982). Contributions of parents and peers to children's moral socialization. Developmental Review, 2, 31-75.

Chinese Culture Connection (1987). Chinese values and the search for culture free dimensions of culture. Journal of Cross-Cultural Psychology, 18, 143-164.

Cohen, J. (1988). Statistical power analysis for the behavioral sciences. Hillsdale, NJ: Lawrence Erlbaum.

Fraser, C., \& Gaskell, G. 1990. The social psychological study of widespread beliefs. Oxford: Clarendon.

Furnham, A. (1988). Lay theories. London: Pergamon.

Grantham, D. (1994). The South in modern America. New York: HarperCollins.

Hofstede, G. (1980). Culture's consequences: International differences in work-related values. Beverly Hills, CA: Sage.

Hu, L., \& Bentler, P. M. (1999). Cutoff criteria for fit indexes in covariance structure analysis: Conventional criteria versus new alternatives. Structural Equation Modeling, 6, 1-55.

Jones, J. M. (1988). Racism in black and white. In P. A. Katz \& D. A. Taylor (Eds.), Eliminating racism (pp. 117-135). New York: Plenum. 
Leung, K., \& Bond, M. H. (2004). Social axioms: A model of social beliefs in multi-cultural perspective. In M. P. Zanna (Ed.), Advances in experimental social psychology. San Diego, CA: Academic.

Leung, K., \& Bond, M. H. (2008). Psycho-logic and eco-logic: Insights from social axiom dimensions. In F. van de Vijver, D. van Hemert, \& Y. H. Poortinga (Eds.), Individuals and cultures in multilevel analysis (pp. 197-219). Mahwah, NJ: Erlbaum.

Leung, K., Bond, M. H., Reimel de Carrasquel, S., Muñoz, C., Hernández, M., Murakami, F., Yamaguchi, S., Bierbrauer, G., \& Singelis, T. (2002). Social axioms: The search for universal dimensions of general beliefs about how the world functions. Journal of Cross-Cultural Psychology, 33, 286-302.

Majors, R., \& Mancini-Billson, J. (1993). Cool pose: The dilemmas of black manhood in America. New York: Touchstone Books.

Markus, H. R., \& Kitayama, S. (1991). Culture and the self: Implications for cognition, emotion, and motivation. Psychological Review, 98, 224-253.

Oyserman, D., Coon, H. M., \& Kemmelmeier, M. (2002). Rethinking individualism and collectivism: Evaluation of theoretical assumptions and meta-analyses. Psychological Bulletin, $128,3-73$.

Reed, J. (1972). The enduring South: Subcultural persistence in mass society. Lexington, MA: Lexington Books.

Reed, J. (1983). Southerners: The social psychology of sectionalism. Chapel Hill, NC: University of North Carolina Press.

Schwartz, S. H. (1992). The universal content and structure of values: Theoretical advances and empirical tests in 20 countries. In M. Zanna (Ed.), Advances in experimental social psychology (vol. 25, pp. 1-65). New York: Academic.

Schwartz, S. H. (1994). Beyond individualism/collectivism: New dimensions of values. In Kim, U., Triandis, H. C., Kagitcibasi, C., Choi, S. C., \& Yoon, G. (1994). Individualism and collectivism: Theory, method, and applications. Newbury Park, CA: Sage.

Singelis, T. M. (1994). The measurement of independent and interdependent self-construals. Personality and Social Psychology Bulletin, 20, 580-591.

Singelis, T. M., \& Brown, W. J. (1995). Culture, self, and collectivist communication: Linking culture to individual behavior. Human Communication Research, 21, 354-389.

Singelis, T. M., Hubbard, C., Her, P., \& An, S. (2003). Convergent validation of the Social Axioms Survey. Personality and Individual Differences, 34, 269-282.

Singelis, T. M., Triandis, H.C. Bhawuk, D., \& Gelfand, M. (1995). Horizontal and vertical dimensions of individualism and collectivism: A theoretical and measurement refinement. Cross-Cultural Research, 29, 240-275.

Slote, W., \& De Vos, G. (Eds.) (1998). Confucianism and the family. New York: State University of New York Press.

Spector, P. E. (1982). Behavior in organizations as a function of employee's locus of control. Psychological Bulletin, 91, 482-497.

Triandis, H. C. (1994). Culture and social behavior. New York: McGraw-Hill.

Triandis, H. C. (1995). Individualism and collectivism. Boulder, CO: Westview.

Vandello, J., \& Cohen, D. (1999). Patterns of individualism and collectivism across the United States. Journal of Personality and Social Psychology, 77, 279-292.

Wrightsman, L. S. (1992). Assumptions about human nature. Newbury Park, CA: Sage. 